\title{
Cariotipo de Leptodactylus insularum (Anura: leptodactilidae (Barbour, 1906) presente en un fragmento de bosque seco tropical de la región caribe colombiana
}

\section{Karyotype of Leptodactylus Insularum (Anura: leptodactylidae (Barbour, 1906) present in a fragment of tropical dry forest of the colombian caribbean region}

GONZÁLEZ T. HENRY ${ }^{1 *}$ M.Sc, CORREA M. MILEIDYS ${ }^{2}$ M.Sc, ÁRQUEZ M. MOISÉS ${ }^{1}$ M.Sc, BELLO L. YESIT ${ }^{2}$ Biol, MORENO R. ALBERTO ${ }^{2}$ M.Sc.

${ }^{1}$ Universidad Simón Bolívar, Colombia. Universidad del Atlántico, Colombia.

Keywords:

Karyotype, chromosome, genetic variation

\section{Palabras Clave:}

Cariotipo, cromosoma, variación genética.

INFORMACIÓN

Recibido: 11-02-2016;

Aceptado: 18-06-2016.

Correspondencia autor:

hgonzalez11@unisimonbolivar.edu.co

\begin{abstract}
Colombia is one of the most biodiverse countries in anuran species, however, many of them are in a vulnerable situation. This has the added problem that for most of them the genetic wealth and possible variations between biotopes is unknown. The aim of this study was to characterize the karyotype of Leptodactylus insularum present in a fragment of tropical dry forest of the Caribbean region (Colombia). Was used the technique described for SCHMIDT (1978) and for chromosomal classification parameters used LEVAN et al. (1964). L. insularum presented a modal number of $n=11$ and $2 n=22$, with chromosome formula $M+4 S M+s M+5 M$. This result varies slightly with respect to the formula of $L$. insularum present in the Andean region (Colombia) which is $2 \mathrm{M}+\mathrm{SM}+\mathrm{M}+\mathrm{ST}+\mathrm{M}+\mathrm{SM}+4 \mathrm{M}$, however, these differences may be associated with biogeographical aspects, so to stay physically separate these populations could have accumulated structural changes in their chromosomes through possible insertion or deletion events. An important aspect is that the specimens that make up this genre generally have a similar chromosome number.
\end{abstract}

\section{Resumen}

Colombia es uno de los países con mayor biodiversidad en especies de anuros, sin embargo, muchos de ellos se encuentran en una situación vulnerable. Esta situación tiene el agravante que para la mayoría de los mismos se desconoce la riqueza genética y las posibles variaciones entre biotopos. El objetivo del presente trabajo fue caracterizar el cariotipo de Leptodactylus insularum presente en un fragmento de BsT la región Caribe (Colombia). Para realizar esto se utilizó técnica de SCHIMID (1978) y para la clasificación cromosomal se utilizó los parámetros de LEVAN et al. (1964). $L$. insularum presentó un numero modal de $n=11$ y $2 n=22$, con formula cromosómica $M+4 S M+s M+5 M$. Este resultado varia con respecto a la fórmula del $L$. insularum presente en la región Andina (Colombia) la cual es $2 \mathrm{M}+\mathrm{SM}+\mathrm{M}+\mathrm{ST}+\mathrm{M}+\mathrm{SM}+4 \mathrm{M}$, sin embargo, estas diferencias pueden estar asociadas a aspectos biogeográficos, por lo que al mantenerse separadas físicamente estas poblaciones, podrían haber acumulado mutaciones estructurales en sus cromosomas mediante posibles eventos de inserción o deleción. Un aspecto importante es que los especímenes que componen este género presentan de forma general un similar número cromosómico. 


\section{Introducción}

Colombia es uno de los países neotropicales que gracias a su amplia variedad de ambientes producto de la interacción de procesos bióticos y abióticos. Presenta un gran número de vertebrados, ocupando el segundo lugar a nivel mundial en anfibios presentes en su territorio; algunos autores sugieren que tal diversidad es una respuesta ante factores como la pluviosidad, la complejidad geográfica y la posición del país, los cuales han generado una amplia gama de hábitats óptimos para el desarrollo de esta fauna (ACOSTA, 2000).

Los anuros son organismos importantes como componentes biológicos, quienes debido a su condición ectodérmica, permeabilidad integumentaria, requerimientos ecológicos que los hace muy dependientes de las condiciones ambientales y extremadamente sensibles a cambios en su hábitat (CASTRO y KATTAN, 1991). Estos elementos que en conjunto con aspectos biogeográficos y de endemismo pueden crear una vulnerabilidad de su existencia. Situación que es preocupante dado que en muchos casos se desconoce su pool genético, desde lo cromosómico hasta lo molecular.

Las especies del género Leptodactylus se caracterizan por poseer 22 cromosomas dentro de los que existe ligeras diferencias entre la morfología de los mismo, siendo dependiente de la especie (AMARO et al., 2006; SALAS et al., 2014). La mayoría de los estudios básicos citogenéticos solo han caracterizado la compasión y estructura cromosómica, es decir, el número diploide y las características morfológicas de los cromosomas mediante el uso de la técnica de coloración convencional Giemsa (AMARO et al., 2004). Además, los estudios en citogenética para Colombia son muy escasos, lo que no permite involucrar planes de manejo a nivel de especies o en poblaciones, desde la perspectiva más amplia de variabilidad genética y de estructura poblacional, siendo estos vacíos en el conocimiento los que en su conjunto constituyen un impedimento para valorar y determinar la diversidad genética existente.

La caracterización citogenética de la especie L. insularum en la región Caribe colombiana, es necesaria ya que esta especie cumple un rol importante en la cadena trófica y en general en el ecosistema donde tiene su hábitat, en especial por presentar un papel ecológico en el control de poblaciones de insectos $u$ otros invertebrados (BOGART, 1979). Más aun cuando, específicamente en anfibios y para el caso de Suramérica, los estudios en citogenética son muy escasos, cobrando especial relevancia en Colombia que es el segundo país con mayor riqueza de anfibios con 733 especies (RUEDA, 2000) y con un mayor número de especies únicas, después de Brasil. Sin embargo, paradójicamente, ocupa el primer puesto en número de especies amenazadas.

El cariotipo de $L$. insularum presente en la región Caribe Colombiana, es un componente clave de referencia para la comprensión de varios aspectos aplicados a la biología básica y complementa la información molecular existente (De SÁ RO et al., 2014) especialmente en su taxonomía y relaciones genéticas con otras especies del género

\section{Materiales y métodos}

Sitio de Muestreo: La captura de los especímenes, se realizó en un área delimitada en cuatro transectos: $11^{\circ} 00^{\prime} 57.67^{\prime \prime} \mathrm{N}$ y $74^{\circ} 52^{\prime} 34.94^{\prime \prime} \mathrm{W}$. La colecta fue manual en jornada nocturna (19:0021:00 hrs). Los especímenes capturados se les tomaron medidas morfométricas con un pie de rey de $0,05 \mathrm{~mm}$ de precisión, haciendo la respectiva anotación de los datos, esto con la finalidad de tener individuos maduros que sobrepasaran la talla de madurez sexual. En total se utilizaron 5 individuos, los cuales fueron identificados positivamente como $L$. insularum en el museo de la Universidad del Atlántico.

Obtención de Cariotipos: Para la obtención del cariotipo de L. insularum, se implementó la técnica de coloración convencional Giemsa, descrita por SCHMID (1978) con modificaciones correspondientes a estandarización de la técnica, en especial con la altura de goteo celular de las muestras, temperatura de calentamiento de las placas, así como la deshidratación previa del citrato de sodio y cloruro de potasio implementadas en las soluciones hipotónicas. 
Los especímenes fueron inyectados intraperitonealmente con una solución de colchicina al $0,1 \%$ por cada $10 \mathrm{~g}$ de peso del animal, cuatro horas antes del sacrificio. Todos los individuos, fueron sacrificados mediante cámara letal de cloroformo; posteriormente se extrajo todo el intestino, el cual fue lavado con una solución de citrato de sodio al $0,09 \%$ durante $10 \mathrm{seg}$.

Seguido, las muestras fueron sometidas a tratamiento hipotónico en $3 \mathrm{~mL}$ de una solución de citrato de sodio al $0,09 \%$ a temperatura ambiente durante $30 \mathrm{~min}$, con una agitación suave de la caja de Petri para evitar que el intestino se doblara. Las muestras fueron fijadas en Carnoy II, posteriormente se raspó suavemente la mucosa intestinal; la suspensión celular resultante se centrifugó a 1000rpm por $7 \mathrm{~min}$, se descartó el sobrenadante y se resuspendió el botón celular con $3 \mathrm{ml}$ de fijador. El paso de centrifugación se realizó por triplicado.

Posterior al centrifugado, el botón celular fue resuspendido en $2 \mathrm{~mL}$ de Carnoy II y goteado sobre portaobjeto limpio, rotulado y precalentado a 60 ${ }^{\circ} \mathrm{C}$, desde una altura 1,8 metros. Los portaobjetos fueron dispuestos en ángulo de $45^{\circ} \mathrm{C}$. A cada portaobjeto se le adicionaron de 3 a 4 gotas de la suspensión celular sobre la lámina portaobjeto dejando secar al aire.

La coloración fue realizada de forma directa sobre las láminas, goteando una solución de Giemsa ( $1 \mathrm{ml}$ de solución comercial $+29 \mathrm{ml}$ de tampón fosfato, $\mathrm{pH} 6,8$ ), hasta un recubrimiento total de la lámina durante 13 minutos, seguidamente lavadas con agua destilada por el envés de la lámina y secadas a temperatura ambiente. Posteriormente se realizaron las observaciones respectivas en el microscopio.

Procesamiento de muestras para microscopía: El microscopio utilizado fue de campo claro, óptico, trinocular, marca Leyca $8 \AA$ modelo CME. Las fotografías fueron realizadas con una cámara Sony ${ }^{\circledR}$ CyberShot $\circledast$ de 12 megapixeles y zoom óptico de $5 x$ de máxima resolución. Las fotografías fueron guardadas como Target Image File ( ${ }^{*}$.tif). Se seleccionaron las mejores metafases. A estas se les suprimió el ruido y elementos sin interés, como artefactos celulares y otras impurezas. Seguidamente con Kario
$2000^{\circledR}$ (ZETA IMAGING y DELTA SISTEMI) los cromosomas fueron organizados por tamaño y posición del centrómero, además a cada cromosoma se le realizo un perfil para medir la longitud total del cromosoma y la longitud de cada brazo, estas medidas se realizaron con el software MicroMeasure $^{\odot}$ (REEVES, 2001).

Análisis de Datos: Los datos de las longitudes fueron guardados en archivo plano. A los mismos, se les realizaron las medidas de tendencia central. Para determinar el número de cromosomas por metafase, se halló el numero modal de $L$. insularum; de igual forma se establecieron los valores de la longitud relativa, índice centromérico, relación entre bazos; estableciéndose la morfología del cromosoma según su índice centromérico de acuerdo a lo descrito por LEVAN et al. (1964), además del cálculo del \%TF (\% de forma total). Para la obtención del ideograma de $L$. insularum se realizó un análisis de histograma de las frecuencias de las longitudes relativas.

\section{Resultados y discusiones}

Los especímenes de L. insularum, objeto de estudio, presentaron en su totalidad muslos fuertemente moteados, presencia de dos líneas dorso laterales de color café que inician a partir de las narinas y puntos negros de manera irregular. Los machos adultos tenían parches centrales de tórax en los tubérculos; con presencia de dos espinas nupciales en cada pulgar, redondeadas y puntiagudas (HEYER y De SÁ RO, 2011), permitiendo establecer que fueron individuos normales. Los especímenes de $L$. insularum, presentaron un numero modal $n=11$ y $2 n=22$ (Figura 1). Para la especie en cuestión todos las muestras analizadas presentaron caracteres morfológicos y morfométricos correspondientes a lo reportado por HEYER y DE SÁ RO (2011) y CUENTAS et al. (2002).

Los cromosomas que constituyen el cariotipo de $L$. insularum, se caracterizaron por presentar tamaños cromosómicos que varían según STEBBINS (1971) entre medianamente grandes $(5-9 \mu)$, y grandes (mayores que $9 \mu$ ). Las longitudes promedios en el presente estudio que oscilan entre $19,08 \pm 0,33 \mu$ hasta $7,2 \pm 0,03 \mu$. Por 


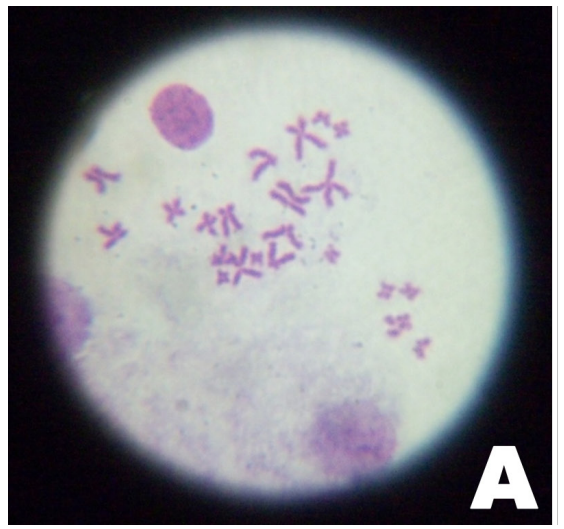

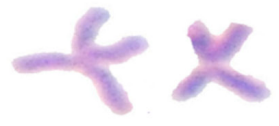

01

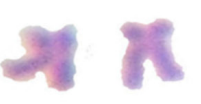

07

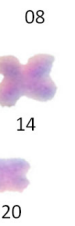

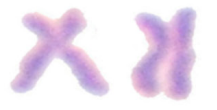

03

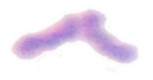

09

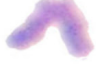

10

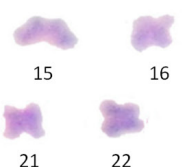

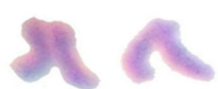

$05 \quad 06$

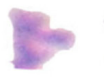

$11 \quad 12$

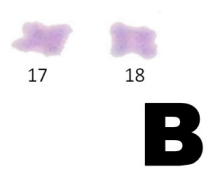

Figura 1. Cromosomas en metafase de L. insularum (A) y Cariotipo de L. insularum colectado en la Región Caribe colombiana (B)

otro lado, la desviación estándar (sd) más alta presento su valor correspondiente al cromosoma \#6 igual a $\pm 0,35 \mu$, entretanto que la más baja se presentó para el cromosoma \#22, $\pm 0,03 \mu$ (Tabla 1), pudiendo estar reflejada esta disparidad ante el hecho de que la diferencia entre los tamaños de los cromosomas se relacione con que el grado de compactación o enrollamiento que puede variar, debido al pre-tratamiento que se le realiza al material para su observación al microscopio, y al margen de error propio del proceso de medición (MAICH y MANERO DE ZUMELZÚ, 2007), la compactación más inmediata puede estar dada para el caso de los cromosomas más pequeños. En el Tabla 1 se detalla la cariometría de los cromosomas de los Individuos de $L$. insularum colectados en la región Caribe colombiana

Durante la metafase, las cromátidas se vuelven más cortas y gruesas, es posible observar el número, tamaño y morfología de los cromosomas en un microscopio óptico tras un tratamiento adecuado de las células (BOGART, 1979); En cuanto a la metodología empleada, para la obtención del cariotipo de L. insularum, se hizo un ajuste en la altura de goteo celular sobre las placas, ya que era necesario un mayor índice de células estalladas para exponer sus cromosomas, altura final fue de 1,80 m. Dado que el choque térmico se aseguró con un precalentamiento de la placa a $60^{\circ} \mathrm{C}$, la placa se inclinó $45^{\circ}$. Los 22 cromosomas fueron alineados acorde con sus respectivas longitudes totales, encontrándose el valor promedio máximo dentro del rango de longitud máxima y mínima registrada para las parejas, a excepción de la desviación estándar que presento valores máximos de $\pm 1,58 \mu$ y mínimos de $\pm 0,53 \mu$.

Los valores de las longitudes relativas de los cromosomas analizados en cada individuo, en conjunto con su promedio y respectiva desviación

Tabla 1. Cariometría de los Cromosomas de los Individuos de L. insularum colectado en la Región Caribe colombiana

\begin{tabular}{|c|c|c|c|c|c|}
\hline Par cromosomal & Longitud total & Longitud relativa & Índice centromérico & $\begin{array}{l}\text { Relación entre } \\
\text { brazos }\end{array}$ & $\begin{array}{l}\text { Clasificación } \\
\text { cromosomica }\end{array}$ \\
\hline Par01 & $18,24 \pm 0,11$ & $13,86 \pm 0,11$ & $48,03 \pm 3,04$ & 1 & $\mathrm{M}$ \\
\hline Par02 & $16,42 \pm 0,09$ & $12,46 \pm 0,09$ & $37,14 \pm 1,13$ & 1,7 & SM \\
\hline Par03 & $14,51 \pm 0,16$ & $11,02 \pm 0,16$ & $34,19 \pm 1,07$ & 2,14 & SM \\
\hline Par04 & $13,21 \pm 0,12$ & $10,02 \pm 0,12$ & $33,99 \pm 0,79$ & 2 & SM \\
\hline Par05 & $12,65 \pm 0,13$ & $9,62 \pm 0,13$ & $33,31 \pm 1,01$ & 2,74 & SM \\
\hline Par06 & $11,33 \pm 0,14$ & $8,60 \pm 0,14$ & $31,41 \pm 2,53$ & 2 & SM \\
\hline Par07 & $10,15 \pm 0,15$ & $7,72 \pm 0,15$ & $44,12 \pm 2,63$ & 1 & $M$ \\
\hline Par08 & $9,09 \pm 0,12$ & $6,90 \pm 0,12$ & $45,34 \pm 1,82$ & 1 & M \\
\hline Par09 & $8,63 \pm 0,09$ & $6,54 \pm 0,09$ & $48,23 \pm 1,55$ & 1 & $\mathrm{M}$ \\
\hline Par10 & $8,61 \pm 0,08$ & $6,24 \pm 0,08$ & $46,66 \pm 3,21$ & 1 & $\mathrm{M}$ \\
\hline Par11 & $7,47 \pm 0,09$ & $5,70 \pm 0,09$ & $47,37 \pm 1,23$ & 1 & $\mathrm{M}$ \\
\hline
\end{tabular}


estándar, presentan los mayores valores de longitud para el individuo 03, sin embargo la diferencia no fue significativa.

Las longitudes relativas del complemento cromosómico, difieren hasta en más de un $50 \%$ entre el tamaño de los cromosomas largos y los cortos, constituyéndose según lo descrito por STEBBINS (1971) en un cariotipo asimétrico quien demuestra ser heterogéneo con marcadas diferencias en el tamaño relativo de los cromosomas del complemento, como consecuencia de reordenamientos cromosómico estructurales. Sin embargo el \% TF para los cromosomas fue de $41 \%$ quien según lo reportado por HUZIWARA (1962), se basa en la relación en porcentaje de la sumatoria total de longitudes de brazos cortos entre la sumatoria total de longitudes cromosómicas totales. Este índice varía entre $0 \%$ y $50 \%$ y en la medida que los valores se acercan al valor máximo mencionado los cariotipos tienden a ser más simétricos; por ende en el presente trabajo los cromosomas tienden a ser simétricos de acuerdo a la posición del centrómero (PERUZZI y EROGLU, 2013).

Se pueden apreciar las longitudes totales de los cromosomas la región centromérica y el índice centromérico de cada par cromosómico en los 5 individuos analizados, pudiéndose observar el mayor índice centromérico en un 48,02 \% de longitud del primer par cromosomal, y el menor fue para el par 6 con un $31,40 \%$.

Las 25 metafases analizadas de los individuos estudiados, registraron un numero modal $n=11$ y $2 n=22$, y en cuanto a las correspondientes metafases todas presentaron morfologías (metacéntricas y submetacéntricos) como se muestra en la Figura 2.

L. insularum, presento un cariotipo caracterizado por un primer par metacéntrico grande, los pares 2, 3, 4 y 6 son submetacéntricos grandes, teniendo la particularidad el par 5 que posee una morfología submetacéntrica menor al no cumplir con el criterio de relación entre brazos, descrito por LEVAN et al. (1964), en cuanto que el par cromosómico 7 es metacéntrico grande, y los pares $8,9,10$ y 11 son metacéntricos medianamente grandes; de forma general el complemento cromosómico presento un $\%$ TF de $41 \%$. Por otro lado, no se detectó

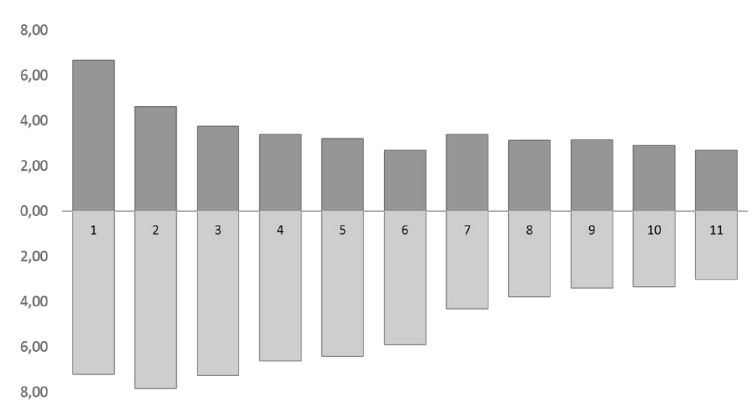

10,00

Figura 2. Ideograma del cariotipo de L. insularum, colectados en la Región Caribe colombiana

heteromorfismo cromosomal en las metafases analizadas.

En contraste con los valores reportados en el Tabla 1, el ideograma del cariotipo de $L$. insularum, colectados en la región Caribe colombiana (Figura 2), refleja las regiones centroméricas de mayor tamaño para los pares Cromosómicos 2, 3, 4, 5 y 6 , entretanto que las regiones de menor tamaño las presentaron los pares cromosómicos 1, 7, 8, 9,10 y 11 presentando la región centromérica una proporcionalidad con la longitud del cromosoma, partiendo que se encuentran en orden de longitud. Estableciéndose la fórmula cromosómica para L.insularum como: $1 \mathrm{M}+4 \mathrm{SM}+1 \mathrm{sM}+5 \mathrm{M}$ de acuerdo al indicen centromérico y los criterios establecidos por LEVAN et al. (1964).

Los eventos de especiación, pueden darse en los organismos gracias a aspectos ambientales, aislamiento reproductivo, la genética, la selección natural, la selección sexual, los cambios cariotípicos, la hibridación, el conflicto genético o los simbiontes intracitoplasmáticos (MABLE, 2011; PERFECTTI, 2009), pero pueden a pesar de este aislamiento guardar características en su formación cariotípica en las que se implican elementos como: número modal, morfología del cromosoma entre otros.

Se puede inferir que la especie en cuestión, de número cromosomal $2 n=22$, y de formula cromosómica: $1 \mathrm{M}+4 \mathrm{SM}+1 \mathrm{sM}+5 \mathrm{M}$, puede no corresponder a lo reportado por BOGART (1974), quien describe en primera instancia el cariotipo de $L$. insularum en la región Caribe colombiana mediante la técnica de coloración convencional 
Giemsa, en el que se establecieron los caracteres morfológicos de los cromosomas, constituyendo un $2 n=22$, sin embargo, las fotografías de los cromosomas de $L$. insularum en la investigación realizada en la región Andina colombiana, no corresponden a un estado de metafase en el que se distingan las hermanas cromátidas, pudiendo estar los cromosomas en un estado de "proface inicial", el cual establece una condición poco diciente de los caracteres morfológicos primordiales (longitud de brazo p y q e índice centromérico); sin embargo, a continuación se establecen las relaciones morfológicas del cariotipo descrito por BOGART (1974), en la región Andina, y las descripciones morfológicas del cariotipo de $L$. insularum para la región Caribe, lo cual evidencia que son la misma especie pero grupos poblacionales distintos. En el Tabla 2 se hace la comparación de la morfología cromosómica por par cromosomal de cada población por regiones biogeográficas.

Tabla 2. Comparación de la morfología cromosómica por par cromosomal de cada población por regiones biogeográficas.

\begin{tabular}{|c|c|c|c|c|c|c|c|c|c|c|c|}
\hline \multirow{2}{*}{$\begin{array}{c}\text { Grupo } \\
\text { poblacional }\end{array}$} & \multicolumn{11}{|c|}{ Par cromosomal } \\
\hline & 01 & 02 & 03 & 04 & 05 & 06 & 07 & 08 & 09 & 10 & 11 \\
\hline $\begin{array}{l}\text { L. insularum } \\
\text { (Región Andina) }\end{array}$ & $M$ & M & SM & $M$ & ST & $M$ & SM & $1 \mathrm{M}$ & M & $M$ & M \\
\hline $\begin{array}{c}\text { L. insularum } \\
\text { (Región Caribe) }\end{array}$ & $M$ & SM & SM & SM & sM & SM & $1 \mathrm{M}$ & M & M & M & M \\
\hline
\end{tabular}

Ambos estudios coinciden en describir un $n=11$ constituido en su mayoría por cromosomas metacéntricos y submetacéntricos, diferenciándose en la existencia de un par cromosómico subtelocéntrico (par 5), quien para el caso de los especímenes de la región Caribe colombiana, este par corresponde a una morfología submetacéntrica menor, al no cumplir con el criterio de relación entre brazos descrito por LEVAN et al. (1964), otra diferencia radica en los pares cromosómicos (2, 4, 6 y 7); estableciéndose una formula cariotípica de $L$. insularum, en el departamento del Atlántico, diferente a la reportada para la región Andina en 1974. Tales diferencias pueden estar asociadas a aspectos biogeográficos, por lo que al mantenerse separadas físicamente estas poblaciones, podrían haberse acumulado mutaciones estructurales en sus cromosomas mediante posibles eventos de inserción o deleción.

De acuerdo a lo reportado por AMARO et al. (2004) para las especies L. ocellatus, L. knudseni y L. pentadactylus, capturados en diferentes zonas de Brasil, presentan relación con $L$. insularum, en cuanto a la ausencia de dimorfismo cromosómico, además de presentar los cromosomas del primer par metacéntrico, 2, 3 y 4 submetacéntricos, 9, 10 y 11 metacéntricos, difiriendo con $L$. insularum, en los pares 5 y 6 metacéntricos, 7 y 8 submetacéntricos en todos los especímenes; divergiendo de lo reportado por BOGART (1974) para el caso de L. ocellatus y L. pentadactylus, $L$. insularum como fue anteriormente descrito. Por otro lado, según el reporte de AMARO et al. (2006) que presenta el cariotipo de L. gracilis, L. mystacinus, $L$. petersii, $L$. pustulatus, $L$. macrosternum, $L$. ocellatus, $L$. labyrinthicus con un $2 n=22$ y L. silvanimbus con un cariotipo distintivo con $2 n=24$, sin embargo, con excepción del primer par cromosomal y la presencia de un par cromosómico más, coincide en presentar los pares Cromosomales 2 al 6 como submetacéntricos y el resto de pares ( 7 al 12) como metacéntricos en relación a $L$. insularum; Por ende, en gran parte los especímenes que componen este género presentan de forma general un similar número cromosómico, además de la ausencia de dimorfismo cromosómico, habiendo solo algunas variaciones morfológicas entre especies.

Sin embargo estas relaciones continúan presentándose para especies de otros géneros de la familia, como lo es el caso de 5 , quien presenta un cariotipo con un $2 \mathrm{n}=22$ bajo la fórmula cariotípica de: $1 \mathrm{M}+3 \mathrm{SM}+2 \mathrm{M}+2 \mathrm{SM}+2 \mathrm{M}$ (SALAS y MARTINO, 2007) que de no ser por presentar los pares cromosómicos 8 y 9 submetacéntricos y los pares 5 y 6 metacéntricos, mostraría una misma fórmula cariotipica en relación a $L$. insularum, más aun cuando este último presenta longitudes relativas de $13,86 \mu \mathrm{m}$ para el primer par cromosómico y $5,7 \mu \mathrm{m}$ para el último par cromosómico. O. cordobae presenta longitudes de $14,09 \mu \mathrm{m}$ para el primer par y $3,53 \mu \mathrm{m}$ para el último par.

No obstante, estas similitudes del cariotipo, no siempre se presentan entre las especies de los distintos géneros de la familia 
Leptodactylidae, como lo es el caso descrito por CUEVAS y FORMAS (2002), quienes describen el cariotipo de Telmatobius philipii del norte de Chile (Leptodactylidae), con un cariotipo constituido por un $n=13$, y morfologías cromosómicas (metacéntricas, submetacéntricas y subtelocéntricas), presentando diferencias claras en la presencia de un $n$ cromosómico distinto al de $L$. insularum, y la presencia de dos pares cromosómico subtelocentricos (par 4 y 5); sin embargo, L. insularum en su 5 to par cromosómico presenta una morfología submecentrica menor, que para el grupo Fuscus es subtelocentrica y característico del grupo.

\section{Referencias}

ACOSTA-GALVIS, A.R. 2000. Ranas, Salamandras y Caecilias. (Tetrapoda:Amphibia) de Colombia. Revista Biota Colombia 1(3):289-319.

AMARO-GHILARDI, R.; RODRIGUES, M.; YONENAGA-YASSUDA, Y. 2004. Chromosomal studies after differential staining and fluorescence in situ hybridization using telomeric probe in three Leptodactylus species (Leptodactylidae, Anura). Caryologia 57(1):53-65.

AMARO, R.C.; SKUK, G.; RAFAEL, O. DE SÁ; TREFAUT, M.; YONENAGA, Y. 2006. Karyotypes of eight species of Leptodactylus (Anura, Leptodactylidae) with a description of a new karyotype for the genus. Phyllomedusa 5(2):119-33.

BOGART, J.P. 1979.Implications of polyploidy in amphibians and reptiles. Basic Life Sci. 13:341-78.

BOGART, J.P.A. 1974. karyosystematic study of frogs in the genus Leptodactylus (Anura: Leptodactylidae). Copeia 3:728-37.

CASTRO, H.F.; KATTAN, G.H. 1991. Estado de conocimiento y conservación de los anfibios del Valle del Cauca. Memorias Primer Simposio Nacional de fauna del Valle del Cauca INCIVA. Cali, Colombia.

CUEVAS, C.; FORMAS, J.R: 2002. Telmatobius philippii, una nueva especie de rana acuática de Ollagüe, norte de Chile (Leptodactylidae). Revista chilena de historia natural 75(1):245-258.

CUENTAS, D.; BORJA, R.; LYNCH, J.D.; RENJIFO, J.M. 2002. Anuros del departamento del Atlántico y Norte de Bolívar. Editor Cencys. Colombia.

De SÁ, R.O.; GRANT, T.; CAMARGO, A.; HEYER, W.R.; PONSSA, M.L.; STANLEY, E. 2014. Systematics of the neotropical genus Leptodactylus Fitzinger, 1826 (Anura: Leptodactylidae): phylogeny, the relevance of non-molecular evidence, and species accounts. South American Journal of Herpetology 9(s1):S1-S100.

HEYER, W.R, De SÁ RO. 2011. Variation, Systematics, and Relationships of the Leptodactylus bolivianus Complex (Amphibia: Anura: Leptodactylidae). Smithsonian Contributions to Zoology 635:1-58.

HUZIWARA, Y. 1962. Karyotype Analysis in Some Genera of Compositae. VIII. Further Studies on the Chromosomes of Aster. American Journal of Botany: 49:116-119.

LEVAN, A.; FREDGA, K.; SANDBERG, A.A. 1964. Nomenclature for centromeric position on chromosomes. Hereditas 52(2):201-220. 
MABLE, B.K.; ALEXANDROU, M.A.; TAYLOR, M.I. 2011. Genome duplication in amphibians and fish: An extended synthesis. Journal of Zoology:284:151-182

MAICH, R.; MANERO DE ZUMELZÚ, D. 2007. Índice Meiótico en Triticale Hexaploide: Análisis y Corrección . BAG. Journal of basic and applied genetics . scieloar 12:23-7.

PERFECTTI, F. 2009. Especiación: Modos y Mecanismos. In: Soler M, editor. Evolución: La Base de la Biología. Proyecto Sur de Ediciones, S.L. Santiago, Chile.

PERUZZI, L.; EROGLU, H. 2013. Karyotype asymmetry: again, how to measure and what to measure? Comparative cytogenetics 7(1):1-9.

REEVES, A. 2001. MicroMeasure: a new computer program for the collection and analysis of cytogenetic data. Genome 44(3):439-443.

RUEDA, ALMONACID J. V. 2000. La Herpetofauna de los Bosques de Florencia Caldas-Una aproximación a su composición, diversidad y relaciones ecológicas. Corporación Autónoma Regional de CaldasSubdirección de Normatización y Calidad Ambiental-CORPOCALDAS. Bogota, Colombia.

SALAS, N.E.; VALETTI, J.A.; GRENAT, P.R.; OTERO, M.A.; MARTINO, A.L. 2014. Meiotic behavior of two polyploid species of genus Pleurodema (Anura: Leiuperidae) from central Argentina. Acta Herpetol 9(1):109-13.

SALAS, N.; MARTINO A.L. 2007. Cariotipo de Odontophrynus cordobae Martino y Sinsch, 2002 (Anura, Leptodactylidae). BAG J basic Appl Genet 18(1):1-5.

SCHMID, M. 1978. Chromosome banding in Amphibia. Chromosoma 66(4):361-388.

STEBBINS G. L. 1971. Chromosomal Evolution in Higher Plants. Addison-Wesley. USA.

ZETA IMAGING Y DELTA SISTEMI. KARIO [Internet]. Roma, It: Delta Sistemi; 2000. 